\title{
Chemical genetics suggests a critical role for lysyl oxidase in zebrafish notochord morphogenesis $\dagger$
}

\author{
Carrie Anderson, $\dagger^{a}$ Stephen J. Bartlett, $\varphi^{b}$ John M. Gansner, ${ }^{c}$ Duncan Wilson, ${ }^{a}$ Ling He, ${ }^{a}$ Jonathan D. Gitlin, ${ }^{c}$ \\ Robert N. Kelsh*a and James Dowden ${ }^{* b d}$
}

Received 21st September 2006, Accepted 30th October 2006

First published as an Advance Article on the web 14th November 2006

DOI: $10.1039 / \mathrm{b} 613673 \mathrm{~g}$

As a result of a chemical genetic screen for modulators of metalloprotease activity, we report that 2-mercaptopyridine- $N$-oxide induces a conspicuous undulating notochord defect in zebrafish embryos, a phenocopy of the leviathan mutant. The location of the chemically-induced wavy notochord correlated with the timing of application, thus defining a narrow chemical sensitivity window during segmentation stages. Microscopic observations revealed that notochord undulations appeared during the phase of notochord cell vacuolation and notochord elongation. Notochord cells become swollen as well as disorganized, while electron microscopy revealed disrupted organization of collagen fibrils in the surrounding sheath. We demonstrate by assay in zebrafish extracts that 2-mercaptopyridine- $N$-oxide inhibits lysyl oxidase. Thus, we provide insight into notochord morphogenesis and reveal novel compounds for lysyl oxidase inhibition. Taken together, these data underline the utility of small molecules for elucidating the dynamic mechanisms of early morphogenesis and provide a potential explanation for the recently established role of copper in zebrafish notochord formation.

\section{Introduction}

Deciphering the orchestrated patterning of cells to form mature functional tissues is a significant challenge. Although widespread genetic screens are informative, the static nature of such mutations militates against interrogating the dynamic processes of tissue development. Small molecules are ideally suited to this task because they offer rapid onset of action and concentration-dependent effects that may be reversed by simple wash-out from the experimental milieu. Consequently, there has been an upsurge of interest in evaluating compound arrays on intact model organisms with a renewed imperative to characterise their targets at the molecular level. ${ }^{1}$

Embryos of the zebrafish Danio rerio are popular model organisms for developmental biologists because they grow ex utero, are transparent and ideally sized for microscopic observation. Large scale random mutagenesis studies have categorised a range of possible mutant morphologies, ${ }^{2-4}$ but

${ }^{a}$ Centre for Regenerative Medicine, Department of Biology \&

Biochemistry, University of Bath, Bath, UK BA2 $7 A Y$.

E-mail: bssrnk@bath.ac.uk; Fax: +44 (0)1225 386 779;

Tel: +44 (0) 1225363828

${ }^{b}$ Wolfson Laboratory of Medicinal Chemistry, Department of Pharmacy and Pharmacology, University of Bath, Bath, UK BA2 7AY

${ }^{c}$ Edward Mallinckrodt Department of Pediatrics, Washington University School of Medicine, 660 South Euclid Ave, St. Louis, Missouri, 63110, USA

${ }^{d}$ School of Chemistry, University of Nottingham, University Park, Nottingham, UK NG7 2RD. E-mail: james.dowden@nottingham.ac.uk; Fax: +44 (0)115 9513 566; Tel: +44 (0)1159513 565

+ Electronic supplementary information (ESI) available: Figure showing the of effect of $\mathbf{5}$ and $\mathbf{6}$ on the notochord and experimental details for compounds 2-6. See DOI: 10.1039/b613673g

\$ These authors have contributed equally and are to be considered joint first authors. characterisation of the underlying biological mechanisms remains a continuing goal. The utility of the chemical approach toward this problem was confirmed when individual embryos subjected to discrete members of an array of approximately one thousand compounds developed morphologies that resembled some reported to arise from random mutagenesis screens. ${ }^{5}$ Subsequent variation of the exposure period for one particular compound provided some insight into the timing of otolith formation. Further chemical genetic studies have been reported in zebrafish and other organisms, including elegant approaches to interrogate functional outcomes of specific gene products. ${ }^{6-10}$ Although prospecting for novel chemical inducers of individual phenotypes has tremendous utility in its own right, the challenge of achieving both chemical and biological definition of the processes that lead to the patterning of cells into tissues and their eventual coordination as a complete organism remains significant.

The notochord is an essential tissue that has both structural and patterning roles. ${ }^{11}$ As a patterning tissue, early notochord signals in both dorsoventral and mediolateral planes influence cell-fate and patterning in the CNS and somites. Structurally, the notochord functions as a skeletal tissue in the embryo and early larva, since it forms a stiffened rod against which muscular contraction can drive motility. Stiffening seems to depend upon two key features: secretion of fibrous collagens, especially collagen II, and vacuolation of notochord cells. ${ }^{12}$ The collagen forms an encasing sheath, thought to constrain expanding notochord cells as their vacuoles inflate, thus generating a hydrostatic pressure that drives the elongation, stiffening and straightening of the notochord. ${ }^{12}$ However, this mechanism has not been thoroughly tested.

Notochord cells are specified during gastrulation, beginning around 6 hours post-fertilisation (hpf), and come to lie in the 
midline. By $24 \mathrm{hpf}$ their vacuoles are expanding and straightening of the trunk and tail is underway. The multiple functions of the notochord are reflected in the abundance and diversity of relevant mutants identified in large-scale mutagenesis screens. ${ }^{13,14}$ Thus, mutations affecting specification of early notochord cells have profound defects on body patterning. ${ }^{15-17}$ Another larger class of mutants affect notochord differentiation and structural roles, thus bashful, grumpy, sneezy and happy are all very shortened and have unvacuolated notochord cells. Molecular characterisation of these and related loci reveal that they are defective in either secretion or notochord sheath components. ${ }^{18-20}$ While these mutants have been characterised molecularly, another class affecting notochord morphogenesis remains uncloned. Amongst these are leviathan, crash test dummy, gulliver and quasimodo ${ }^{13,14}$ which, in contrast to sleepy and related mutants, display welldifferentiated notochord cells, but with a violently distorted arrangement in both dorsomedial and mediolateral planes. Importantly, whilst gulliver and quasimodo mutants show additional characteristics, leviathan and crash test dummy show only a notochord defect. These latter mutants were isolated in separate screens and may well be allelic.

In the course of a study aimed at examining the role of zincdependent matrix metalloproteases (MMPs) in zebrafish, we tested small molecule modulators that might provide precise and specific control over metal binding biological targets. This paper describes the utility of one compound, 2-mercaptopyridine- $N$-oxide (MCP) (1, Fig. 1), that potently induces an undulating notochord phenotype reminiscent of the known mutants leviathan and crash test dummy. ${ }^{13,14}$ Varied timing and duration of compound application furnished precise control over the position and length of the notochord undulations and insight into the timing of notochord morphogenesis. Detailed analysis of the undulating notochord lead to the nomination of the copper dependent lysyl oxidase [Lox, EC 1.4.3.13] as a putative biological target of MCP 1. Additivity for irreversible Lox inhibitor $\beta$-APN ${ }^{21}$ and MCP 1 in an assay for oxidase activity in zebrafish lysates offers strong support for this hypothesis. Together these observations provide a satisfying

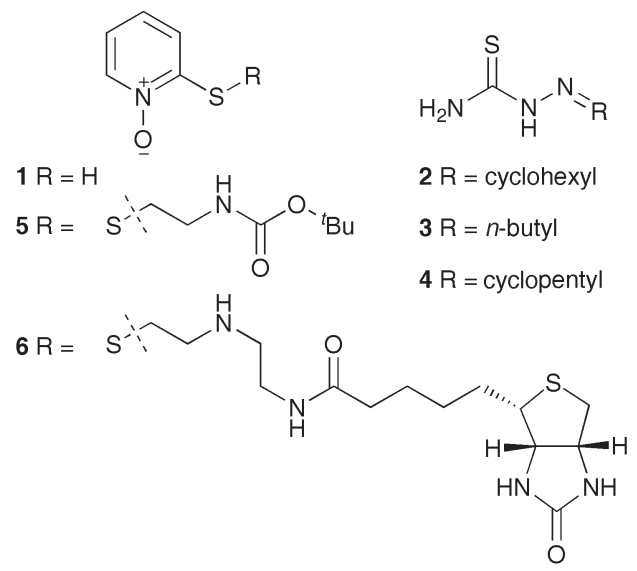

Fig. 1 Structures of active compounds. 2-Mercaptopyridine- $N$-oxide $\mathbf{1}$ and sulfur derivatives (4 and $\mathbf{5}$ ), as well as thiosemicarbazones derived from cyclohexanone $\mathbf{2}, n$-butanal $\mathbf{3}$ and cyclopentanone $\mathbf{4}$ are shown. explanation for the undulating notochord phenotype recently reported in a zebrafish model for Menkes disease. ${ }^{22}$

\section{Results}

\section{Metal binding compounds evaluated}

We selected compounds, such as matrix metalloprotease (MMP) inhibitors, that might be elaborated to achieve specific control over individual metal-bearing biological targets and thus to examine the target's potential role in cell migration or remodelling of the extracellular matrix (ECM). Compounds containing a hydroxamic acid motif have been widely utilised as inhibitors of MMPs and other metal binding proteins. ${ }^{23} \mathrm{~A}$ small number of commercially-available hydroxamate compounds were evaluated, but these had no apparent effect on zebrafish embryo development. We next set out to find simple binding motifs that might deliver greater potency and bioavailability than hydroxamic acid derivatives. Puerta and co-workers recently compared eleven zinc-binding groups for potency against MMP-3, from which MCP 1 emerged as by far the strongest inhibitor $\left(\mathrm{IC}_{50} \sim 35 \mu \mathrm{M}\right) .{ }^{24}$ We therefore sought to evaluate its effect on zebrafish development. Embryos treated with MCP $1(100 \mathrm{nM})$ produced a phenotype with distinct notochord undulations that closely resembled leviathan and crash test dummy mutants identified in largescale mutagenesis screens (Fig. 2). ${ }^{13,14}$

\section{Notochord defects induced over a wide concentration range}

Embryos exposed to 2-mercaptopyridine- $N$-oxide 1 displayed pronounced notochord deformations in both dorsoventral and mediolateral planes at concentrations between 100 and $500 \mathrm{nM}$ (Fig. 2B). Under these conditions embryos otherwise looked rather normal, although deformations of myotomes and spinal cord accompanied the gross distortions of the notochord. Lower doses $(50 \mathrm{nM})$ induced weaker notochord deformations, whereas higher doses of MCP $1(100 \mu \mathrm{M})$ generated an absence of melanisation in addition to the notochord defect at both 48 and 72 hpf (Fig. 3j,k). In comparison, untreated siblings showed strong melanisation of both neural crestderived melanophores in the body and the pigmented retinal epithelium of the eye (Fig. 3a,b). Intermediate doses of around $5 \mu \mathrm{M}$ produced weaker pigmentation defects in keeping with the normal melanisation observed at lower doses (Fig. 31). Thus high doses lead to a compound phenotype of notochord deformation and impaired pigmentation. We confirmed that embryos treated with these high doses of MCP $\mathbf{1}$ are true albinos, lacking melanin, but retaining melanocytes, by in situ hybridisation with the melanocyte marker, dopachrome tautomerase. $^{25}$ These studies confirmed the presence of nonmelanised melanocytes (data not shown). Albinism is associated with decreased activity of tyrosinase (EC 1.14.18.1), a copper-dependent enzyme, for which metalbinding compounds have been reported as inhibitors. ${ }^{26}$

An earlier phenotypic screen ${ }^{5}$ had reported thiosemicarbazide derivatives that induced similar effects, thus we sought to compare MCP 1 with a small number of such compounds derived from cyclohexanone $\mathbf{2}$, butanal $\mathbf{3}$ and cyclopentanone 4 (Fig. 1). $100 \mu \mathrm{M}$ doses of each compound (2-4) all resulted in 


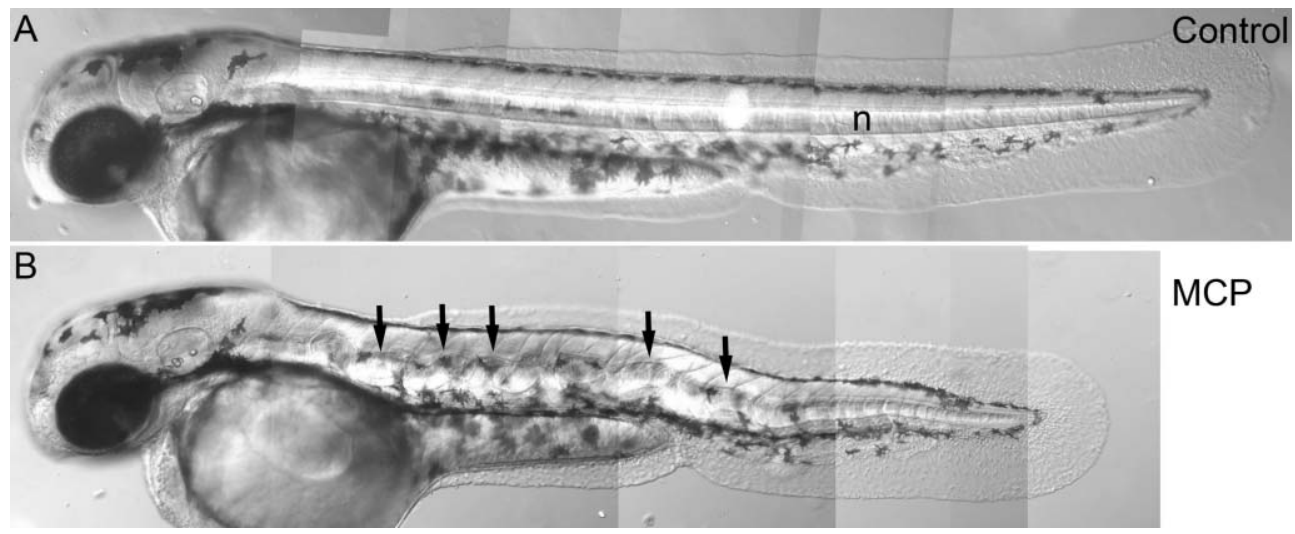

Fig. 2 MCP 1-induced notochord deformation. Lateral views of $52 \mathrm{hpf}$ wild-type embryos after treatment with $100 \mathrm{nM}$ MCP 1 . Note the prominent undulating deformations (B) of the notochord (n), contrasting with straight morphology of untreated sibling control (A).
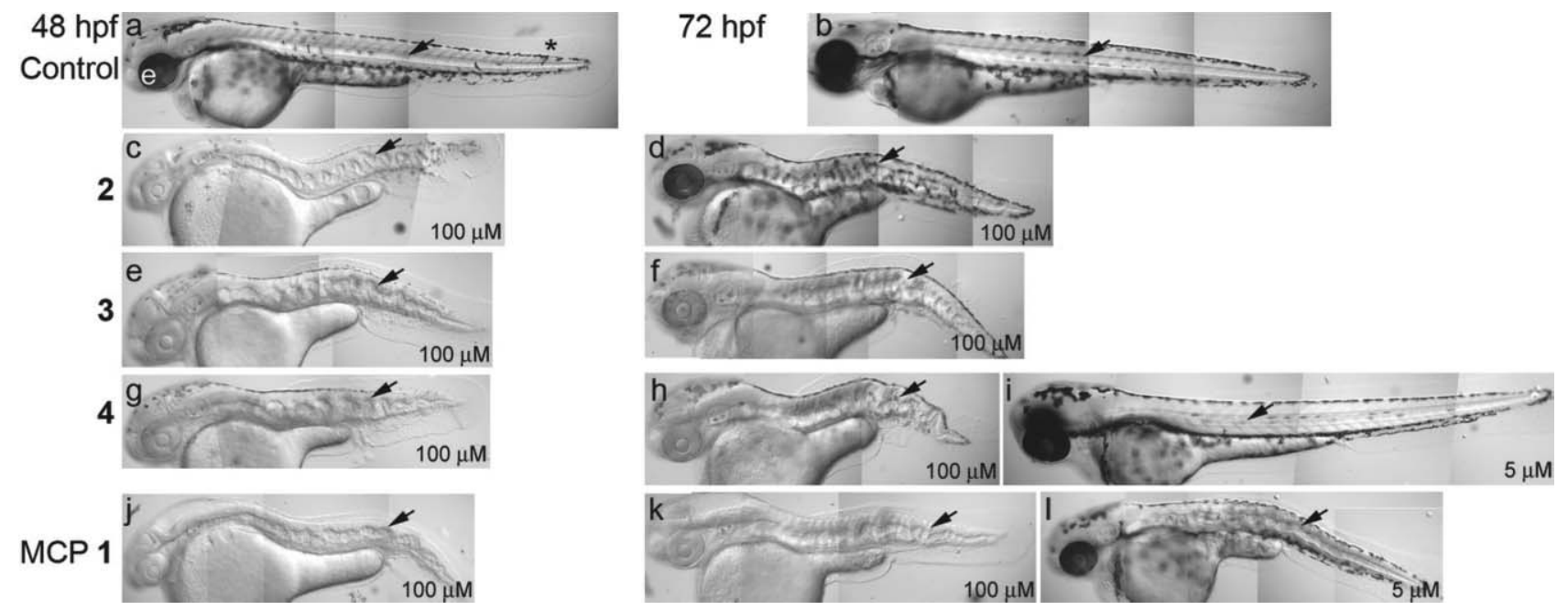

Fig. 3 MCP 1-induced notochord and pigmentation phenotypes. Lateral views of 48 (a,c,e,g,j) and 72 hpf (b,d,f,h,k,i,l) embryos after treatment with thiosemicarbazones derived from cyclohexanone $\mathbf{2}(\mathrm{c}, \mathrm{d})$, butanal 3 (e,f), cyclopentanone $\mathbf{4}$ (g-i) or MCP $\mathbf{1}$ (j-1) or untreated controls (a,b). All treated embryos were exposed to $100 \mu \mathrm{M}$ of the compound, except (i) and (1) where the dose was only $5 \mu \mathrm{M}$. Note pronounced notochord (arrow) deformation induced by all $100 \mu \mathrm{M}$ treatments and by $5 \mu \mathrm{M}$ MCP $\mathbf{1}$, whereas lower dose of $\mathbf{2}, \mathbf{3}$ and $\mathbf{4}$ usually resulted in normal notochord development (shown for 4 (i), but similar for others). Note reduced melanisation of eyes (e) and melanocytes $(*)$ under high dose treatments, with $48 \mathrm{hpf}$ treated embryos usually lacking or having very reduced melanin (c,e,g,j), but with melanisation recovering at $72 \mathrm{hpf}$ after treatment with $100 \mu \mathrm{M} 2$ (d), 3 (f), or 4 (h). Treatment with $5 \mu \mathrm{M} \mathrm{2-4}$ does not affect melanisation at $72 \mathrm{hpf}$ (shown for 4 (i), but similar for others). MCP 1 treatment results in a stronger melanisation phenotype, with little or no recovery at $72 \mathrm{hpf}$ in $100 \mu \mathrm{M}(\mathrm{k})$ and moderate recovery at $72 \mathrm{hpf}$ in $5 \mu \mathrm{M}$ (l) treatment.

dramatic notochord deformation and pronounced albinism at 48 and $72 \mathrm{hpf}$ (Fig. 3c-h). Although there were consistent differences in the degree of albinism induced at this concentration, each was weaker in effect at $72 \mathrm{hpf}$ than MCP 1 (Fig. 3k). At lower ( $5 \mu \mathrm{M})$ doses, all thiosemicarbazide variants gave embryos of normal pigmentation at $72 \mathrm{hpf}$ (Fig. 3i) and, interestingly, approximately 95\% of them had normal notochords, with the remainder displaying only weak abnormalities that were most pronounced in the tail. In distinct contrast, MCP 1 treatment at this same concentration gave consistent strong notochord defects, as well as a mild melanisation defect (Fig. 31).

Simple sequestration of metal ions is a potential mode of action for these compounds that might explain these observations. This was tested by exploring supplementation of the embryo buffer with dissolved zinc $\left(\mathrm{ZnCl}_{2}\right)$ or copper $\left(\mathrm{CuCl}_{2}\right)$ ions in the presence of MCP 1 (100-50 $000 \mathrm{nM}$, Table 1). No abrogation of the notochord defect was observed in the presence of either zinc chloride (up to $400 \mathrm{nM}$ ), or copper chloride (up to $1 \mu \mathrm{M}$ ), although embryonic lethality precluded assessment of notochord phenotype at higher doses of both MCP 1 and copper. Interestingly, the melanisation defect seen only at high doses of MCP was partly abrogated by $100 \mathrm{nM}$ copper. The notochord phenotype induced by 100 nM MCP 1 remained unaffected even when $25 \mu \mathrm{M}$ copper chloride supplementation was used.

MCP 1 precisely phenocopies recessive lethal single locus mutations, and its effects are not rescued by copper supplementation. We therefore propose that MCP 1 inhibits a single biological target at low concentrations. At higher concentrations, a second target, probably tyrosinase, is affected which, unlike the notochord defect, may be the result 
Table 1 Effects of $\mathrm{CuCl}_{2}$ supplementation on MCP 1-induced notochord deformation and albinism ${ }^{a, b}$

\begin{tabular}{|c|c|c|c|c|c|c|}
\hline \multirow[b]{2}{*}[\mathrm{MCP}]{$/ \mathrm{nM}$} & \multirow[b]{2}{*}[\mathrm{CuCl}_{2}]{$/ \mathrm{nM}$} & \multirow[b]{2}{*}{$N$} & \multicolumn{2}{|l|}{ Notochord } & \multicolumn{2}{|c|}{ Melanisation } \\
\hline & & & $\%$ Wavy (\%) & Normal (\%) & Albino (\%) & Normal (\%) \\
\hline \multirow[t]{3}{*}{0} & 0 & 21 & 0 & 100 & 0 & 100 \\
\hline & 100 & 20 & 0 & 100 & 0 & 100 \\
\hline & 1000 & 19 & 0 & 100 & 0 & 100 \\
\hline \multirow[t]{3}{*}{100} & 0 & 20 & 100 & 0 & 0 & 100 \\
\hline & 100 & 20 & 100 & 0 & 0 & 100 \\
\hline & 1000 & 18 & 100 & 0 & 0 & 100 \\
\hline \multirow[t]{3}{*}{500} & 0 & 20 & 100 & 0 & 0 & 100 \\
\hline & 100 & 4 & 100 & 0 & 0 & 100 \\
\hline & 1000 & 0 & $\mathrm{X}^{c}$ & $\mathrm{X}^{c}$ & $\mathrm{X}^{c}$ & $\mathrm{X}^{c}$ \\
\hline \multirow{3}{*}{1000} & 0 & 19 & 100 & 0 & 0 & 100 \\
\hline & 100 & 8 & 100 & 0 & $75^{d}$ & 25 \\
\hline & 1000 & 0 & $\mathrm{X}^{c}$ & $\mathrm{X}^{c}$ & $\mathrm{X}^{c}$ & $\mathrm{X}^{c}$ \\
\hline \multirow[t]{3}{*}{50000} & 0 & 20 & 100 & 0 & 100 & 0 \\
\hline & 100 & 16 & 100 & 0 & 100 & 0 \\
\hline & 1000 & 0 & $\mathrm{X}^{c}$ & $\mathrm{X}^{c}$ & $X^{c}$ & $\mathrm{X}^{c}$ \\
\hline
\end{tabular}

${ }^{a}$ Supplementation with $\mathrm{ZnCl}_{2}$ gave equivalent results, data not shown. ${ }^{b} \mathrm{CuCl}_{2}$ alone does not induce notochord deformations. ${ }^{c}$ Lethal at high concentrations. ${ }^{d}$ A mild decrease in pigmentation was observed. $N=$ number of embryos scored.

of copper depletion. We therefore set out to define more precisely the timing and effect of 2-mercaptopyridine- $N$-oxide $\mathbf{1}$ at low concentrations, in order to try to identify a likely target biomolecule.

\section{Specific timing of notochord defect}

Variation of the timing and duration of application of chemical modulators is a powerful method of interrogating their mechanism of action. Variation in the time of application and withdrawal of 2-mercaptopyridine- $N$-oxide $\mathbf{1}$ from the embryo buffer showed that MCP 1 had no effect either during early $(0.75$ to $6 \mathrm{hpf})$ or late $(>24 \mathrm{hpf})$ stages, thus defining a narrow temporal window during which the notochord is vulnerable to chemically induced deformation. Further experiments revealed correlation between MCP 1 application and the position of the start of the notochord defect (Fig. 4). Thus, embryos treated from early in this sensitive phase (12 hpf)
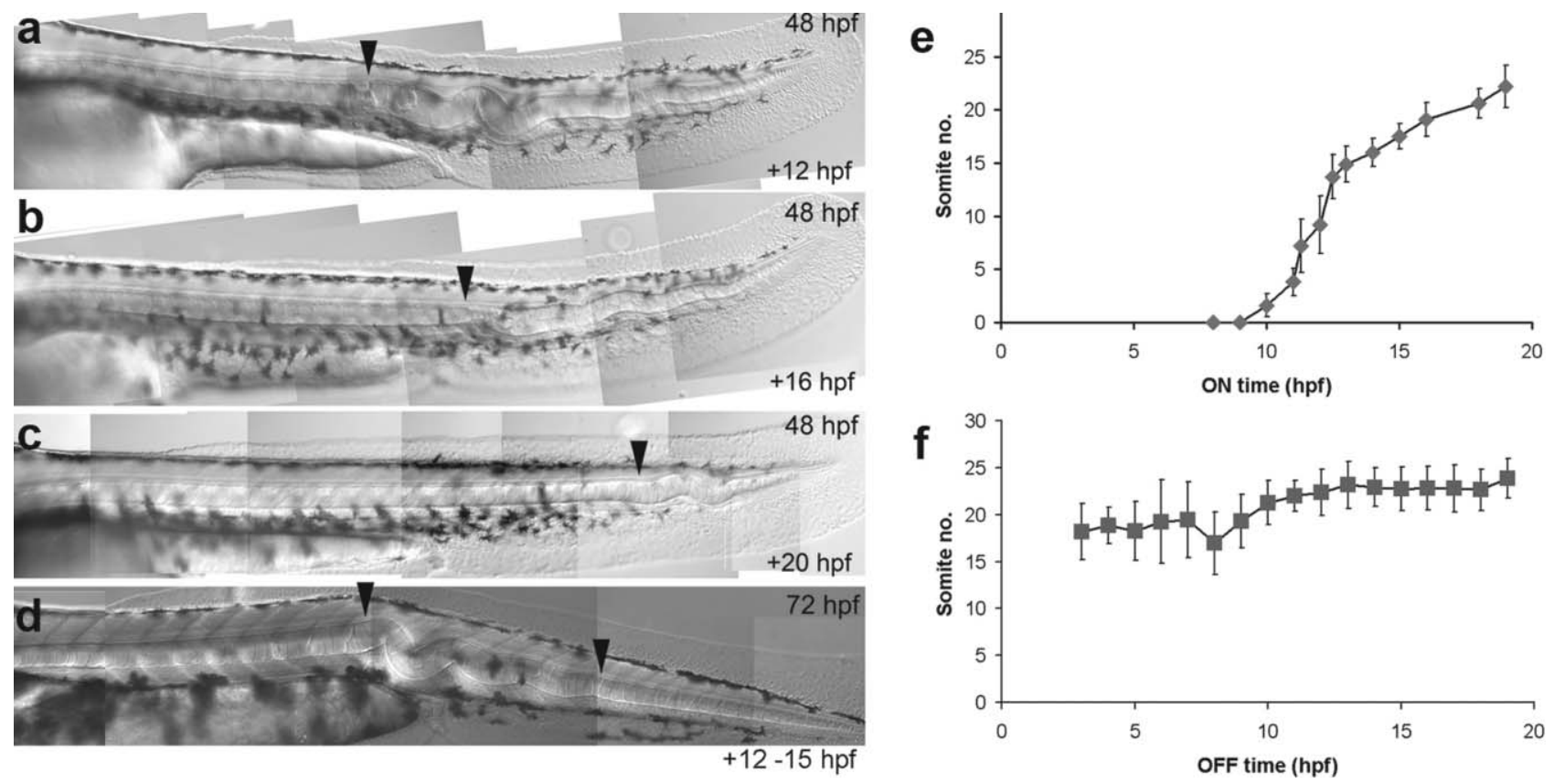

Fig. 4 Temporal control of notochord morphogenesis. The anterior boundary of the notochord defect (arrowhead) shifts in the posterior direction as the time of MCP 1 addition is delayed. $200 \mathrm{nM}$ MCP 1 was added at (a) 12 hpf, (b) 16 hpf and (c) 20 hpf; all embryos were photographed at $48 \mathrm{hpf}$. (d) The relationship between the time of MCP 1 addition and the anterior boundary of the notochord defect is given as the adjacent somite number (mean \pm s.d.). Embryos here were scored at $3 \mathrm{dpf}$. $N$ for each time-point $>10$. (e) If MCP 1 added for a short time interval $(12-15 \mathrm{hpf})$ and then removed, notochord defects are localised within restricted zone (demarcated by arrowheads), here shown in $3 \mathrm{dpf}$ embryo. (f) If MCP 1 was present before $6 \mathrm{hpf}$, then removed at various subsequent time-points, the posterior boundary of the affected notochord region was shifted, although the rate of change was lower than for 'ON graph'. $N$ for each time-point $>15$. 

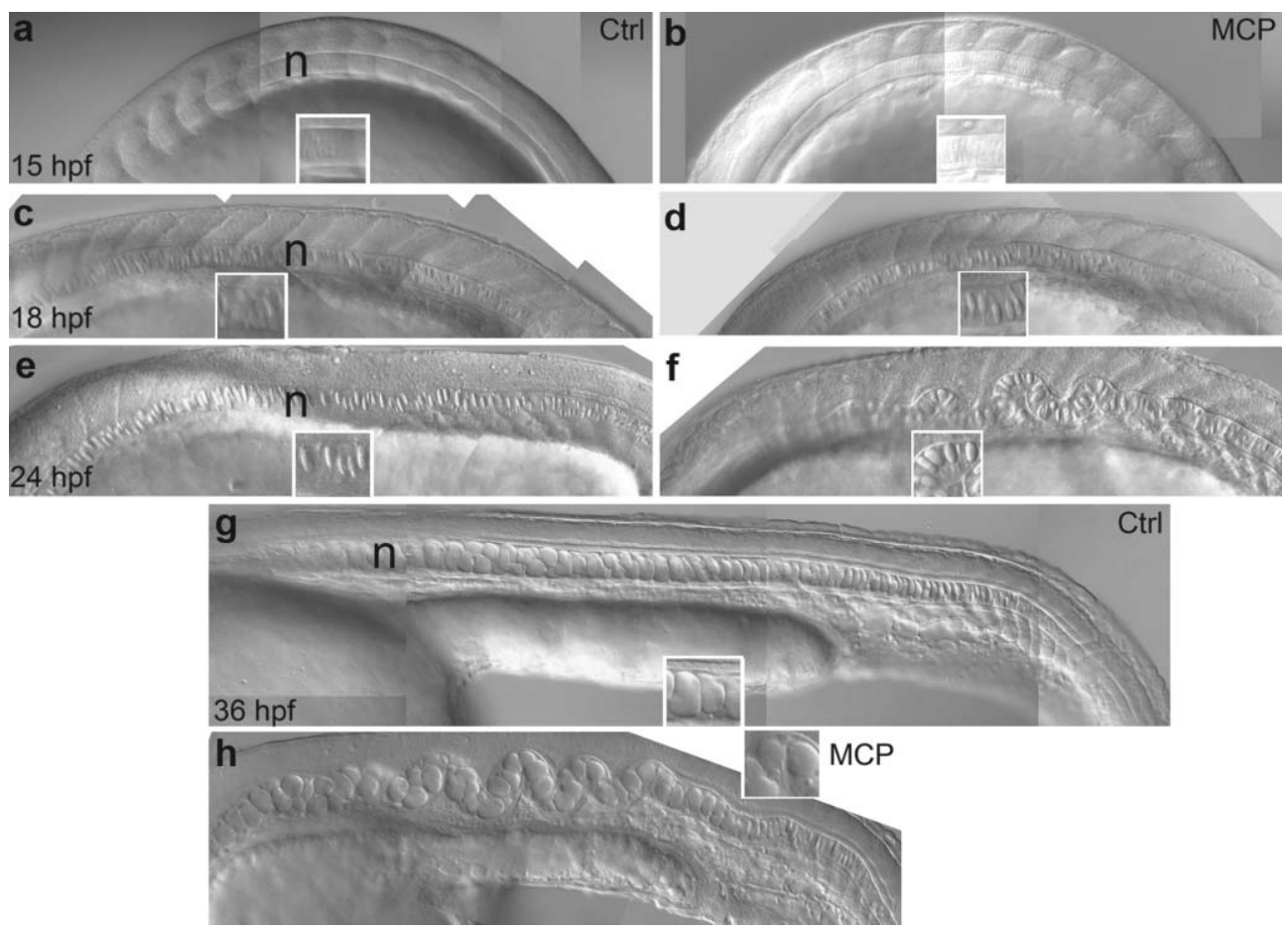

Fig. 5 MCP 1 affects notochord morphology from late-somitogenesis stages. Lateral views of trunk of embryos treated with 200 nM MCP 1 $(\mathrm{b}, \mathrm{d}, \mathrm{f}, \mathrm{h})$ or left untreated (a,c,e,g) and photographed at 15 (a,b), 18 (c,d), 24 (e,f) and 36 (g,h) hpf. Insets show close-ups of notochord cells to show progressive enlargement of vacuoles. The notochord (n) appears rather normal at early stages, but rapidly becomes severely kinked during the phase of cell vacuolation (just detectable at $18 \mathrm{hpf}$ (d), dramatic by $24 \mathrm{hpf}(\mathrm{f})$ ). Note in (g) and (h) the clear correlation between notochord distortion and incomplete rostrocaudal expansion.

showed notochord defects in the posterior trunk and throughout the tail, whereas treatment from 16 hpf affected only the tail and treatment from $20 \mathrm{hpf}$ only the posterior tail (Fig. 4a-c). Quantitative analysis of this effect, using the position of the anteriormost notochord deformation, confirmed a linear, biphasic spatial-temporal response (' $\mathrm{ON}$ ' graph; Fig. 4e). We observed a steeper gradient during the early phase and shallower gradient for the later phase, apparently corresponding to rapid development of the trunk regions followed by slower development of the tail.

Restoration of the normal, straight notochord in the posterior tail could be observed when MCP 1 was removed from the growth milieu later in the sensitive period, but there was no rescue of the defect in the anterior tail (Fig. 4d). In contrast to the 'ON' graph (Fig. 4e), there was a poor correlation between the time of MCP 1 removal and the restored tail region. Instead, the posterior boundary of the notochord defect generally shifted slowly caudally as the compound $\mathbf{1}$ was removed at progressively later time-points ('OFF' graph; Fig. 4f). We tested whether MCP 1 was trapped within the chorion, thus slowing its removal, but dechorionation of embryos prior to buffer replacement made little difference to the curve (data not shown). This analysis suggests that notochord development is only vulnerable to MCP $\mathbf{1}$ treatment during a precise phase of differentiation and that each region of the notochord responds independently to its effects. The latter point is emphasised by addition of MCP $\mathbf{1}$ at $12 \mathrm{hpf}$, followed by removal at $15 \mathrm{hpf}$ ('OFF-ON-OFF'), which produced embryos with normal-looking straight notochord in the trunk and posterior tail, but with a severely-undulating zone in the anterior tail (Fig. 4d).

We next explored how early notochord deformation becomes visible, and whether this correlates to specific phases of notochord development. In untreated embryos, notochord cell vacuolation (differentiation) is the main process observed during 15-36 hpf stages (Fig. 5). Vacuoles first became prominent around $18 \mathrm{hpf}$, were clearly expanded by $24 \mathrm{hpf}$ and fully-formed by $36 \mathrm{hpf}$ (Fig. 5a,c,e,g). Embryos treated with MCP 1 also show vacuolation throughout this period with an apparently normal progression of notochord cell differentiation (Fig. 5b,d,f,h). Interestingly, chemically (1) treated fish have normal morphology at the beginning of this phase (Fig. 5b) and deformities only first appear in the middle phase. In contrast to the straight notochord of untreated fish, there is already a hint of the undulated morphology by $18 \mathrm{hpf}$, which becomes very prominent by $24 \mathrm{hpf}$ (Fig. 5e,f). Thus, the MCP 1 sensitivity window does not correlate with notochord cell specification. Instead, deformation occurs early during notochord differentiation just prior to vacuolation, apparently by affecting the maintenance of notochord morphology as vacuolation drives its expansion.

\section{Thiol derivatives of 1 retain activity}

Two 2-mercaptopyridine- $N$-oxide derivatives, one derived from $N$-(tert-butoxycarbonyl)-2-bromoethylamine $\mathbf{5}$, and the other containing a biotin tag 6 (Fig. 1) were generated with a view to future target molecule isolation using the biotinylated 
compound 6. All compounds induced the same notochord defect as the parent compound, but with different potencies (see Fig. S1 in the ESI $\dagger$ ). Thus, MCP 1 was fully effective at $200 \mathrm{nM}$, whereas each variant showed somewhat reduced activity (5 was effective down to $2 \mu \mathrm{M}$; and $\mathbf{6}$ only down to $20 \mu \mathrm{M})$. This difference presumably arises from the different binding affinities at the biological target, although differences in bioavailability cannot be ruled out.

Differential interference contrast (DIC) microscopy of embryos treated with MCP 1 and these derivatives provided detailed images of the early stage notochord that revealed intriguing differences in notochord cell morphology. Thus, untreated siblings showed a distinct 'stack of pennies' arrangement of notochord cells, whereas equivalent chemically treated (1) cells were spherical, and more loosely organised (Fig. S1b-i in the ESI $\dagger$ ).

\section{2-Mercaptopyridine- $\mathrm{N}$-oxide 1 subtly affects notochord sheath differentiation}

In all cases, chemically-induced notochord undulations were accompanied by a shortened rostrocaudal body axis. Neither notochord cell number (mock treated mean \pm s.d. count, $115.6 \pm 12.3(n=5), 100 \mu \mathrm{M}$ MCP 1 treated, $108.6 \pm 7.06$ $(n=5))$ nor somite number (mock-treated, $33.2 \pm 1.92(n=5)$, $100 \mu \mathrm{M}$ MCP 1 treated, $33.0 \pm 2.24(n=5))$ at $25-30 \mathrm{hpf}$ were affected by MCP 1 treatment, arguing that notochord distortions were not the result of changed proliferation nor impaired axis growth. Normal notochord formation proceeds with volume expansion of the component cells being constrained by the notochord sheath in all planes except for elongation, ultimately producing an extended stiff rod. ${ }^{27}$ The highly-vacuolated but more-spherical appearance of chemically treated notochord cells suggested decreased constraint from the notochord sheath.

We investigated expression of collagen II (Col2 $\alpha 1)$, a major mechanical component of the notochord sheath, ${ }^{28}$ in chemically treated embryos. Whole-mount in situ hybridisation analysis revealed remarkable differences. Whereas normal embryos display transient mRNA transcription of col $2 \alpha 1$, largely down-regulated by $24 \mathrm{hpf}$ (see Fig. 6a where notochord expression is mostly seen in the tail-tip), embryos exposed to 2-mercaptopyridine- $N$-oxide $\mathbf{1}$ showed ectopic expression in much of the notochord (Fig. 6b,d). This might indicate that the MCP 1 inhibits a feedback mechanism that down-regulates collagen expression.

We used transmission electron microscopy to analyse notochord sheath structure more directly. Transverse sections through the 23 and $27 \mathrm{hpf}$ control fish revealed a regular structure with an inner layer of circularly arranged collagen fibres forming the medial layer, surrounded by the outer layer of rostrocaudally-arranged fibres (Fig. 6e,g). Importantly, chemically-treated fish clearly showed collagen fibres in an approximation to this arrangement, however the collagen fibres were somewhat disorganised so that these two layers were less clear (Fig. 6f,h). These observations suggest that, whilst a collagen sheath is formed, its structural integrity may be compromised by the presence of 2-mercaptopyridine- $N$-oxide $\mathbf{1}$.

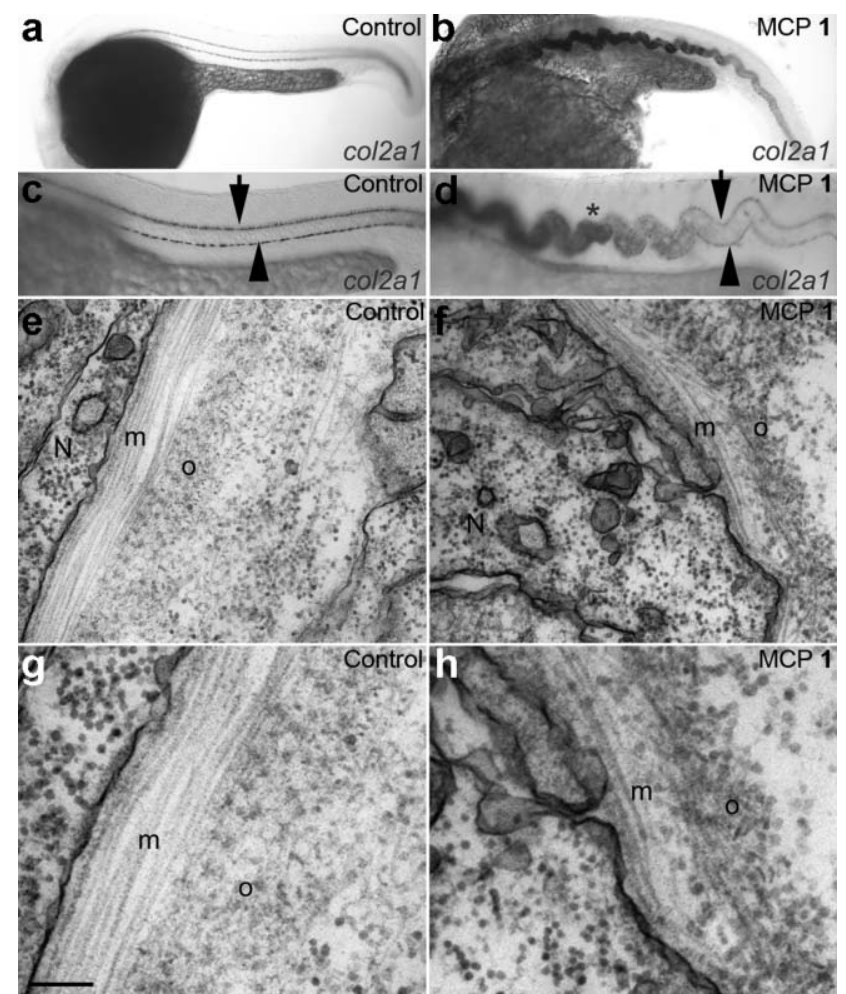

Fig. 6 MCP 1 treatment disrupts notochord sheath differentiation. (a-d) col2 $\alpha 1$ is expressed ectopically in the notochord of MCP 1-treated embryos. (a,c) Lateral views of untreated embryo at $24 \mathrm{hpf}$ show lack of expression of col $2 \alpha 1$ in notochord throughout trunk and most of tail, contrasting with strong expression in floorplate (arrow) and hypochord (arrowhead). (b,d) Sibling embryo treated with $100 \mu \mathrm{M}$ MCP 1 shows strong ectopic expression of col2 $\alpha 1$ in trunk notochord $\left(^{*}\right)$, as well as floorplate and hypochord expression. Identical phenotypes were seen with 2,3 and 4 (data not shown). (e-h) Transmission electron micrographs of notochord sheath in $27 \mathrm{hpf}$ control (e,g) and MCP 1-treated (100 nM, f,h) embryos. Note regular organization of collagen fibres in medial (m) and outer (o) layers of normal sheath, and disorganized fibre arrangements in MCP 1-treated fish. N: notochord cell. Scale bar, $200 \mathrm{~nm}(\mathrm{e}, \mathrm{f}) ; 100 \mathrm{~nm}(\mathrm{~g}, \mathrm{~h})$.

\section{2-Mercaptopyridine- $\mathrm{N}$-oxide 1 inhibits lysyl oxidase}

Organisation of collagen fibres is achieved by crosslinking between aldehyde functional groups generated by the copper containing enzyme lysyl oxidase [Lox, EC 1.4.3.13] from the $\varepsilon$-amine of collagen lysine residues. Good numbers of collagen fibres could be observed in embryos treated with MCP 1, but they were disorganised. We hypothesised that MCP 1 and its derivatives inhibit copper-dependent Lox type enzymes by binding the catalytic domain.

To test this idea, we performed an assay for lysyl oxidase activity in adult zebrafish extracts. ${ }^{29}$ Irreversible lysyl oxidase inhibitor $\beta$-APN (500 $\mu \mathrm{M}$, Fig. 7$){ }^{21}$ was examined first and lysyl oxidase activity was depleted by nearly $80 \%$ (Fig. 7). In the same assay, $5 \mu \mathrm{M}$ MCP 1 reduced lysyl oxidase activity in the fish extracts by around $30 \%$. Interestingly, addition of both compounds resulted in an additive effect, suggesting that the mechanisms of action of $\beta$-APN and MCP 1 were different. Approximately the same amount of inhibition is obtained with $100 \mu \mathrm{M} \beta$-APN as with $5 \mu \mathrm{M}$ MCP 1 (data not shown), 


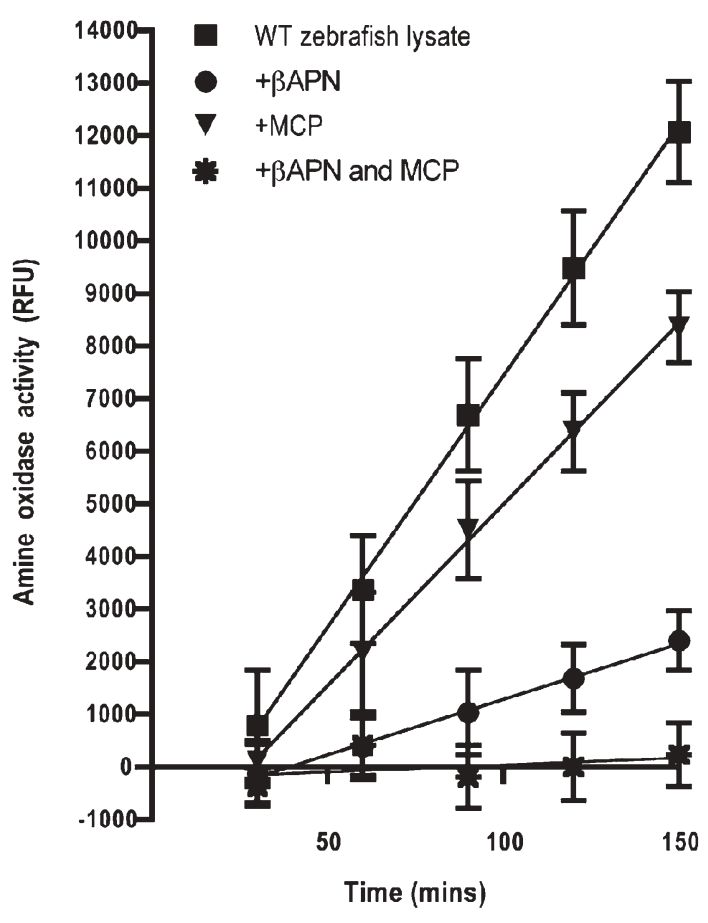

Fig. 7 MCP 1 inhibits lysyl oxidase activity. Lysyl oxidase activity in crude fish extracts is measured in the absence and presence of a known lysyl oxidase inhibitor ( $\beta$-APN) or MCP 1 or both combined. Data shown is representative of three separate experiments and activity is presented in relative fluorescent units with each data point as the mean of three triplicate measurements, error bars indicate standard deviation.

reflecting the greater potency of MCP $\mathbf{1}$ as a lysyl oxidase inhibitor in vitro. Of note, $\beta$-APN causes notochord undulation in wild-type zebrafish embryos at millimolar concentrations (data not shown), confirming that MCP-1 also has superior potency in vivo. Although the fluorometric assay does not distinguish Lox from other amine oxidase activity, in view of all the data presented here we conclude that MCP $\mathbf{1}$ is likely to act via specific inhibition of Lox activity.

\section{Discussion}

We report characterisation of the effects of MCP 1 and related compounds on zebrafish embryos, noting a dramatic undulating notochord morphology that is strongly and specifically reminiscent of leviathan and crash test dummy mutants. This notochord effect is one feature of osteolathyritic phenotypes reported in various toxicological studies in organisms (particularly fish and frogs) exposed to a range of chemicals. ${ }^{28,30-33}$ The zinc complex of MCP 1, a component of anti-dandruff shampoo, had previously been reported as a teratogen in zebrafish and Japanese medaka. ${ }^{34}$ More recently, a thiol derivative of MCP 1 was reported to induce wavy notochord in zebrafish and also compared to the leviathan mutant. ${ }^{35}$ However, neither of these papers provides a detailed mechanistic analysis of this phenotype. Here we show by variation of the timing and duration of embryo exposure to MCP 1 that this effect was reversible, that morphogenesis of the notochord proceeds in a linear fashion and that local notochord morphogenesis proceeds independently of adjacent regions. Furthermore, our analysis has shown that MCP 1 sensitivity corresponds to the phase of notochord differentiation, when notochord cells are expanding by vacuolation. Our data revealed defects in the notochord sheath and pointed toward lysyl oxidase as the target for MCP 1, a hypothesis reinforced by the oxidase assay in zebrafish lysates. Thus, our study establishes MCP-treatment as a useful tool for detailed studies of notochord morphogenesis and osteolathyrism.

The observation that 2-mercaptopyridine- $N$-oxide 1 precisely phenocopies specific recessive lethal single locus mutations suggests that it inhibits a single target compound at low doses. At higher doses, where albinism is induced in addition, a second target, likely tyrosinase itself, is also inhibited. In principle, MCP 1 may induce wavy notochord and albinism phenotypes by a mechanism of generic sequestration of copper, or by specific binding to this metal at the active sites of target cuproproteins. Very recently, Mendelsohn and coworkers proposed a hierarchy of phenotypes arising from a gradient of copper deficiency in zebrafish embryos. ${ }^{22}$ Seven copper binding compounds were reported to cause albinism at low concentrations and a leviathan-like notochord defect at higher concentrations. Interestingly, these effects were abrogated when $\mathrm{CuCl}_{2}(25 \mu \mathrm{M})$, but no other transition metals, was added to a buffer containing these chelators and developing embryos. These effects were attributed to irreversible copper sequestration. This conclusion was strengthened by the observed enhanced phenotype when low doses of the chelators were combined with heterozygous mutations in a copper transport ATPase gene (atp7a). ${ }^{22}$ Thiosemicarbazides, along with many other compounds previously identified as osteolathyrogens, appear to exert their effects by sequestration of copper. Our experiments involving exposure of embryos to thiosemicarbazide derivatives (2-4) reinforces the hierarchy of albinism at lower concentrations $(5 \mu \mathrm{M})$, and wavy notochord at higher $(100 \mu \mathrm{M})$ concentrations proposed by Mendelsohn and colleagues. Significantly, MCP 1 presented the opposite effect, inducing the notochord defect at low concentrations (3-100 $\mathrm{nM}$ ) and albinism only at higher concentrations $(5-100 \mu \mathrm{M})$. Furthermore, supplementing the MCP 1 buffer with $\mathrm{CuCl}_{2}$ did not abrogate the notochord defect, although it did partially rescue the melanisation defect. Finally, the Mendelsohn study revealed a distinct sensitivity window for the copper sequestrators, with even brief treatment at $3 \mathrm{hpf}$ being sufficient to produce the full notochord and albinism phenotype. This window is completely distinct from the MCP-sensitivity window revealed by our studies. Together, this data strongly militates against a sequestration model for MCP 1 action at low concentrations.

Instead, our analysis of the weakened notochord sheath suggested specific inhibition of a target involved in collagen cross-linking. Our assay for Lox activity in zebrafish lysates showed that MCP 1, previously described as a specific MMP3 inhibitor, ${ }^{24}$ in fact more potently targets lysyl oxidase in zebrafish embryos. Furthermore, it appears to do so by a mechanism distinct from that of $\beta$-APN. ${ }^{21}$ The role of copper in the oxidase reaction is not clear, but copper-free bovine lysyl oxidase retains approximately $40 \%$ activity compared to the wild-type (this lower activity being attributed to 
decomposition of the lysine tyrosyl quinone cofactor, LTQ, suggesting that the metal imparts co-factor stability). ${ }^{36}$ MCP 1 may block the active site by strongly binding to copper.

Lysyl oxidases (Lox) are initially expressed as preproenzymes, processed to release a signal protein, then cleaved by bone morphogenetic protein 1 (BMP-1)/Tolloid (TLD)-like metalloproteases to remove the $\mathrm{NH}_{2}$-terminal domain. ${ }^{37,38} \mathrm{We}$ discount such MMPs as potential targets because hydroxamate MMP inhibitors do not affect collagen cross-linking in osteoblastic cell cultures. ${ }^{39}$ Furthermore, developmental studies of BMP-1/TLD-like metalloproteases suggest that organs other than notochord would be affected by their inactivation in zebrafish embryos. ${ }^{40}$

Lysyl oxidases possess a highly conserved catalytic domain, including a copper binding site and proximate lysine and tyrosine residues that ultimately provide the LTQ co-factor. Lox $x^{-1--}$ mouse embryos die perinatally of vascular abnormalities $^{41,42}$ and show disorganised collagen fibrils in the skin and lungs. ${ }^{43}$ Whole mount in situ hybridization studies in Xenopus laevis revealed varied timing of expression and localisation of transcripts for Xlox, Xloxl-1 and Xloxl-3, but each of these appeared in the notochord. Xenopus embryos treated with $\beta$-APN showed a range of effects in addition to undulating notochord, including abnormal gut coiling and edemas, but no defects were apparent in the heart. ${ }^{33}$ MCP 1 treated fish show abnormal myotome, CNS and other defects, although these are generally consistent with secondary defects resulting from the notochord abnormalities. The zebrafish genome sequencing project is ongoing, but at least as many lox-like genes as Xenopus are expected and expression studies have yet to be performed. Nonetheless, the effects of MCP 1 in zebrafish appear rather specific and it will be interesting to establish whether these effects can be attributed to individual lysyl oxidases.

A Xenopus study using $\beta$-APN concluded that the notochord defect was likely to arise late, as a mechanical effect of muscle activity; ${ }^{33}$ the very early onset of the notochord deformations noted in our study indicates that defects appear during early stages of morphogenesis, consistent with the predictions of the unrestrained sheathing model. Evaluation of MCP 1 in Xenopus embryos and comparison to $\beta$-APN treatment will, therefore, be interesting.

\section{Conclusions}

Phenotypic screening is a convenient route to uncover novel chemical modulators, but identification of the biological target and characterisation of its contribution to pathways generating altered morphologies is a continuing challenge. We present an accumulation of evidence arising from a strategy tightly focused around evaluation of a small number of compounds predicted to be target metal binding proteins. This led to the conclusion that MCP 1 induces an undulating notochord phenotype by weakening the notochord sheath, leading to over-vacuolation and disordering of notochord cells. Transmission electron microscopy revealed disrupted collagen fibre cross-links within the notochord sheath, which considered alongside misexpressed col2 $\alpha 1$, suggested lysyl oxidase as the molecular target of MCP 1. Our demonstration that MCP $\mathbf{1}$ has lysyl oxidase inhibitory activity confirms this model and identifies lysyl oxidase as a strong candidate gene for the currently uncloned leviathan and crash test dummy mutants. This paper also reinforces a key advantage arising from the reversibility of compound exposure, which in our studies provided precise control over the timing and location of the notochord phenotype that subsequently informed our understanding of notochord patterning in the developing zebrafish embryo.

Wider functions for lysyl oxidase are emerging, ${ }^{37,38}$ for example, an essential role for hypoxia-induced tumour metastasis was recently reported. ${ }^{44}$ Individual isoforms probably control discrete aspects of this wider functional repertoire, for which isoform-selective chemical probes would be attractive. Confirmation that MCP $\mathbf{1}$ is an inhibitor of lysyl oxidase may inspire development of related compounds that exploit this apparently novel mode of action, although selectivity over other metalloproteins, such as tyrosinase, will be an important design criterion.

\section{Experimental}

\section{Preparation of chemicals}

2-Mercaptopyridine- $N$-oxide $\mathbf{1}$ and $\beta$-aminopropionitrile was purchased from Sigma, all other compounds were synthesised as detailed in the ESI†. Chemicals were dissolved in DMSO (Sigma) and made up to $10 \mathrm{mM}$ stock in 50\% DMSO and 50\% embryo medium (embryo medium $=5 \mathrm{mM} \mathrm{NaCl}, 0.17 \mathrm{mM}$ $\mathrm{KCl}, 0.33 \mathrm{mM} \mathrm{CaCl}_{2}, 0.33 \mathrm{mM} \mathrm{MgSO}$, 10-5 methylene blue). All chemicals were used at a working dilution (usually around $200 \mathrm{nM}$, as noted) dissolved in 1\% DMSO in embryo medium. Control embryos were placed in embryo medium supplemented with $1 \%$ DMSO; the latter treatment had no discernible effect on embryo development.

\section{Fish husbandry}

Wild-type zebrafish of strain $\mathrm{AB}$ were kept in a dedicated facility at the University of Bath. Embryos were obtained from single pair matings. Approval for all experiments on live animals was obtained from the local ethical review panel where necessary and performed in accordance with all national and local guidelines and regulations.

\section{Chemical treatment}

Synchronised AB wild-type zebrafish embryos were raised in embryo medium until time of treatment. For the 'ON graph', embryo medium was replaced at appropriate time, with $200 \mathrm{nM}$ MCP 1, raised to $48 \mathrm{hpf}$, dechorionated, anaesthetised with Tricaine (Sigma) and scored for the anterior boundary of notochord distortion. Control embryos were placed in embryo medium with $1 \%$ DMSO. For the 'OFF graph', embryos were placed in $200 \mathrm{nM}$ MCP 1 prior to $6 \mathrm{hpf}$, then at appropriate times medium was removed, embryos were rinsed in embryo medium and then placed in fresh embryo medium. Embryos were raised to $48 \mathrm{hpf}$, then were scored for the posterior boundary of the notochord defect. 


\section{Microscopy and photography}

Embryos were viewed under an Eclipse E800 microscope (Nikon) using DIC or fluorescence optics and photographed with a SPOT RT color camera (Diagnostic Instruments, Inc).

\section{Lysyl oxidase assay ${ }^{29}$}

Fish were euthanized and pulverized in liquid nitrogen with a mortar and pestle. The powder was collected in PBS and rocked for $1 \mathrm{~h}$ at $4{ }^{\circ} \mathrm{C}$ before centrifuging at $10000 \mathrm{~g}$ for $30 \mathrm{~min}$ to obtain an insoluble fraction, which was mechanically homogenized and extracted overnight in $6 \mathrm{M}$ urea-50 mM sodium borate $\mathrm{pH} 8.2$ with agitation at $4{ }^{\circ} \mathrm{C}$. The supernatant was isolated after a second centrifugation at $30000 \mathrm{~g}$ for $30 \mathrm{~min}$ and diluted to $1.2 \mathrm{M}$ urea with $50 \mathrm{mM}$ sodium borate, $\mathrm{pH}$ 8.2. Lysates were then concentrated using Amicon YM-10 columns and the flow-through saved.

Assays were carried out in triplicate at $37{ }^{\circ} \mathrm{C}$ using $150 \mu \mathrm{L}$ of lysate or flow-through and $50 \mu \mathrm{L}$ of a $4 \mathrm{x}$ reaction buffer containing $4 \mathrm{U} \mathrm{mL}^{-1}$ type II horseradish peroxidase, $40 \mathrm{mM}$ cadaverine, and $40 \mu \mathrm{M}$ Amplex UltraRed in $1.2 \mathrm{M}$ urea$50 \mathrm{mM}$ sodium borate, $\mathrm{pH}$ 8.2. Amplex UltraRed fluorescence was measured at defined intervals using an excitation of $560 \mathrm{~nm}$ and an emission of $590 \mathrm{~nm}$. For each sample, the background fluorescence of the corresponding flow-through with or without drug was subtracted to generate a corrected fluorescence, reported in relative fluorescent units (RFU).

\section{Acknowledgements}

We thank the Wellcome Trust for a Showcase Award to JD and RNK. JMG was supported by NIH Medical Scientist Training Program grant T32 GM07200. JDG is supported by NIH DK44464.

\section{References}

1 D. R. Spring, Org. Biomol. Chem., 2003, 1, 3867.

2 P. Haffter, M. Granato, M. Brand, M. C. Mullins, M. Hammerschmidt, D. A. Kane, J. Odenthal, F. J. M. van Eeden, Y. J. Jiang, C. P. Heisenberg, R. N. Kelsh, M. FurutaniSeiki, E. Vogelsang, D. Beuchle, U. Schach, C. Fabian and C. Nusslein-Volhard, Development, 1996, 123, 1 .

3 W. Driever, L. Solnica-Krezel, A. F. Schier, S. C. F. Neuhauss, J. Malicki, D. L. Stemple, D. Y. R. Stainier, F. Zwartkruis, S. Abdelilah, Z. Rangini, J. Belak and C. Boggs, Development, 1996, 123, 37.

4 A. Amsterdam, S. Burgess, G. Golling, W. B. Chen, Z. X. Sun, K. Townsend, S. Farrington, M. Haldi and N. Hopkins, Genes Dev., 1999, 13, 2713.

5 R. T. Peterson, B. A. Link, J. E. Dowling and S. L. Schreiber, Proc. Natl. Acad. Sci. U. S. A., 2000, 97, 12965.

6 R. T. Peterson, S. Y. Shaw, T. A. Peterson, D. J. Milan, T. P. Zhong, S. L. Schreiber, C. A. MacRae and M. C. Fishman, Nat. Biotechnol., 2004, 22, 595.

7 H. M. Stern, R. D. Murphey, J. L. Shepard, J. F. Amatruda, C. T. Straub, K. L. Pfaff, G. Weber, J. A. Tallarico, R. W. King and L. I. Zon, Nat. Chem. Biol., 2005, 1, 366.

8 P. E. Bayliss, K. L. Bellavance, G. G. Whitehead, J. M. Abrams, S. Aegerter, H. S. Robbins, D. B. Cowan, M. T. Keating, T. O'Reilly, J. M. Wood, T. M. Roberts and J. Chan, Nat. Chem. Biol., 2006, 2, 265.

9 T. C. Y. Kwok, N. Ricker, R. Fraser, A. W. Chan, A. Burns, E. F. Stanley, P. McCourt, S. R. Cutler and P. J. Roy, Nature, 2006, 441, 91.
10 M. L. Tomlinson, R. A. Field and G. N. Wheeler, Mol. BioSyst., $2005,1,223$

11 D. L. Stemple, Development, 2005, 132, 2503-2512.

12 N. S. Glickman, C. B. Kimmel, M. A. Jones and R. J. Adams, Development, 2003, 130, 873-887.

13 J. Odenthal, P. Haffter, E. Vogelsang, M. Brand, F. J. M. van Eeden, M. Furutani Seiki, M. Granato, M. Hammerschmidt, C. P. Heisenberg, Y. J. Jiang, D. A. Kane, R. N. Kelsh, M. C. Mullins, R. M. Warga, M. L. Allende, E. S. Weinberg and C. Nusslein-Volhard, Development, 1996, 123, 103.

14 D. L. Stemple, L. Solnica-Krezel, F. Zwartkruis, S. C. F. Neuhauss, A. F. Schier, J. Malicki, D. Y. R. Stainier, S. Abdelilah, Z. Rangini, E. Mountcastle Shah and W. Driever, Development, 1996, 123, $117-128$.

15 K. Fekany, Y. Yamanaka, T. C. Leung, H. I. Sirotkin, J. Topczewski, M. A. Gates, M. Hibi, A. Renucci, D. Stemple, A. Radbill, A. F. Schier, W. Driever, T. Hirano, W. S. Talbot and L. Solnica-Krezel, Development, 1999, 126, 1427-1438.

16 A. F. Schier, Curr. Opin. Neurobiol., 1997, 7, 119.

17 W. S. Talbot, B. Trevarrow, M. E. Halpern, A. E. Melby, G. Farr, J. H. Postlethwait, T. Jowett, C. B. Kimmel and D. Kimelman, Nature, 1995, 378, 150.

18 P. Coutinho, M. J. Parsons, K. A. Thomas, E. M. A. Hirst, L. Saude, I. Campos, P. H. Williams and D. L. Stemple, Dev. Cell, 2004, 7, 547.

19 M. J. Parsons, S. M. Pollard, L. Saude, B. Feldman, P. Coutinho, E. M. A. Hirst and D. L. Stemple, Development, 2002, 129, 3137.

20 S. M. Pollard, M. J. Parsons, M. Kamei, R. N. W. Kettleborough, K. A. Thomas, V. N. Pham, M. K. Bae, A. Scott, B. M. Weinstein and D. L. Stemple, Dev. Biol., 2006, 289, 64.

21 S. S. Tang, P. C. Trackman and H. M. Kagan, J. Biol. Chem., 1983, 258, 4331.

22 B. A. Mendelsohn, C. Yin, S. L. Johnson, T. P. Wilm, L. SolnicaKrezel and J. D. Gitlin, Cell Metab., 2006, 4, 155.

23 M. Whittaker, C. D. Floyd, P. Brown and A. J. H. Gearing, Chem. Rev., 1999, 99, 2735.

24 D. T. Puerta, J. A. Lewis and S. M. Cohen, J. Am. Chem. Soc., 2004, 126, 8388.

25 R. N. Kelsh, B. Schmid and J. S. Eisen, Dev. Biol., 2000, 225, 277.

26 R. C. Hider and K. Lerch, Biochem. J., 1989, 257, 289.

27 D. S. Adams, R. Keller and M. A. R. Koehl, Development, 1990, $110,115$.

28 M. A. Haendel, F. Tilton, G. S. Bailey and R. L. Tanguay, Toxicol. Sci., 2004, 81, 390.

29 A. H. Palamakumbura and P. C. Trackman, Anal. Biochem., 2002, 300, 245.

30 J. E. Snawder and J. E. Chambers, Toxicol. Appl. Pharmacol., 1993, 121, 210.

31 D. A. Dawson, B. D. Scott, M. J. Ellenberger, G. Poch and A. C. Rinaldi, Environ. Toxicol. Pharmacol., 2004, 16, 13.

32 C. J. Vanleeuwen, T. Helder and W. Seinen, Aquat. Toxicol., 1986, 9, 147.

33 T. J. Geach and L. Dale, Differentiation, 2005, 73, 414

34 K. Goka, Environ. Res., 1999, 81, 81.

35 S. M. Sternson, J. B. Louca, J. C. Wong and S. L. Schreiber, J. Am. Chem. Soc., 2001, 123, 1740.

36 C. L. Tang and J. P. Klinman, J. Biol. Chem., 2001, 276, 30575.

37 H. M. Kagan and W. D. Li, J. Cell. Biochem., 2003, 88, 660.

38 J. Molnar, K. S. K. Fong, Q. P. He, K. Hayashi, Y. Kim, S. F. T. Fong, B. Fogelgren, K. M. Szauter, A. Mink and K. Csiszar, BBA-Proteins Proteom., 2003, 1647, 220.

39 N. Pischon, H. Babakhanlou-Chase, L. Darbois, W. B. Ho, M. C. Brenner, E. Kessler, A. H. Palamakumbura and P. C. Trackman, J. Cell Physiol., 2005, 203, 111.

40 S. A. Connors, J. A. Tucker and M. C. Mullins, Dev. Biol., 2006, 293, 191.

41 J. M. Maki, J. Rasanen, H. Tikkanen, R. Sormunen, K. Makikallio, K. I. Kivirikko and R. Soininen, Circulation, 2002, 106, 2503

42 I. K. Hornstra, S. Birge, B. Starcher, A. J. Bailey, R. P. Mecham and S. D. Shapiro, J. Biol. Chem., 2003, 278, 14387.

43 J. M. Maki, R. Sormunen, S. Lippo, R. Kaarteenaho-Wiik, R. Soininen and J. Myllyharju, Am. J. Pathol., 2005, 167, 927.

44 J. T. Erler, K. L. Bennewith, M. Nicolau, N. Dornhofer, C. Kong, Q. T. Le, J. T. A. Chi, S. S. Jeffrey and A. J. Giaccia, Nature, 2006 , 440, 1222. 